\title{
Cestóides Trypanorhyncha parasitos de Scianideos de importância comercial, capturados no Litoral Amazônico, Brasil
}

\section{Cestodes (Trypanorhyncha) parasites of Scianids of commercial importance captured in the Amazonian littoral, Brazil}

\author{
Lílian de Nazaré Santos Dias, ${ }^{*}$ Rosildo dos Santos Paiva, ${ }^{* *}$ Sérgio Carmona de São Clemente,,$* \star *$ \\ Alessandra Epifanio Rodrigues, ${ }^{* * * *}$ Augusto Solano Lobo Peralta, ${ }^{* * * *}$ Edílson Rodrigues Matos ${ }^{* * * *}$
}

\begin{abstract}
Resumo
Foram examinados 201 exemplares de peixes das espécies Cynoscion acoupa (Lacepède, 1801), Macrodon ancylodon (Bloch e Scheider, 1801), Plagioscion squanosissimus (Heckel, 1840), todos da família scianidea, no período de agosto de 2006 a dezembro de 2007, com o objetivo de registrar e identificar cestoides da ordem Trypanorhyncha, que parasitam peixes de valor comercial capturados no litoral amazônico. Os peixes foram identificados e mensurados durante a filetagem. Foram examinadas as seguintes regiões do corpo: serosa e as musculaturas dorso-lateral e ventro-abdominal. Os blastócitos de Trypanorhyncha encontrados foram removidos e levados ao Laboratório de Pesquisa Carlos Azevedo da UFRA para sua identificação. Do total de peixes pesquisados 138 (68,65\%) estavam parasitadas por cestoides da ordem Trypanorhyncha, sendo registradas as seguintes espécies de parasitas: Poecilancistrium caryophyllum, Pterobothrium heteracanthum, Pterobothrium crassicolle e Callitetrarhynchus gracilis, sendo $P$. caryophyllum a que apresentou a maior prevalência, ocorrendo em todas as espécies de peixes estudadas. A musculatura dorso-lateral, a de maior valor comercial, foi a região com maiores índices parasitários. A alta taxa de parasitismo mostra a necessidade da realização de estudos epidemiológicos, assim como o desenvolvimento de procedimentos de inspeção e controle das parasitoses em peixes de interesse comercial da região amazônica.
\end{abstract}

Palavras-chave: Trypanorhyncha, inspeção, peixes.

\begin{abstract}
There were evaluated 201 fish specimens belonging to the species Cynoscion acoupa (Lacepède, 1801), Macrodon ancylodon (Bloch \& Scheider, 1801), Plagioscion squanosissimus (Heckel, 1840), all of them members of the family Scianidae, during the period from August of 2006 to December of 2007, with the objective of recording and identifying the cestodes from the order Trypanorhyncha, that parasitize fish of commercial value captured in the Brazilian Amazonian littoral. Fishes were measured, identified and during the filleting process there were examined the following fish body parts: serous membrane and the backlateral and ventral-abdominal musculature. Found blastocysts of Trypanorhyncha were removed and sent to the Laboratory Carlos Azevedo of the UFRA for identification. All the three studied fish species were parasitized by Trypanorhyncha, to a total of 138 (68,65\%) specimens parasitized. The recorded parasites species were: Poecilancistrium caryophyllum, Pterobothrium heteracanthum, Pterobothrium crassicolle and Callitetrarhynchus gracilis. Poecilancistrium caryophyllum showed the highest prevalence, occurring on all studied species. The back-lateral musculature, which has the highest commercial value, was the body part affected by the highest parasitism levels. The high parasitism level show the necessity of conducting epidemiological studies, as well as the development of procedures for inspection and parasitic control on fish of commercial interest from the Amazonian region.
\end{abstract}

Keywords: Trypanorhyncha, fish, inspection.

\footnotetext{
* Universidade Federal do Pará, Curso de Pós-Graduação em Ciência Animal / UFPA. Autor para correspondência: Conjunto Satélite, WE-09, nº 1276 B, CEP: 66670-250 - Tel. (91) 81756530 - 91903901; email: vetdias12@yahoo.com.br

** Universidade Federal Pará.

*** Universidade Federal Fluminense.

**** Universidade Federal Rural da Amazônia.

***** Agência de Defesa Agropecuária do Pará.
} 


\section{Introdução}

A família Scianidae é constituída por peixes comumente encontrados em águas rasas da plataforma continental sobre fundo de areia e lamas (Menezes e Figueiredo,1980). São de grande interesse comercial para a região amazônica e representa uma das principais famílias de pescado mais consumidas no estado do Pará. No que se refere à saúde pública, a ordem Trypanorhyncha não possui potencial zoonótico, porém adquirem importância higiênica, pelo aspecto repugnante que ocasionam quando os peixes apresentam elevada intensidade destes parasitas na musculatura, peritônio, mesentério e fígado. Levando ao impedimento de sua comercialização pela fiscalização sanitária ou rejeitada pelo consumidor, acarretando perdas et al. (1991). A identificação das espécies de cestoides baseou-se nos trabalhos de Dollfus (1942), Rego (1987), Carvajal e Rego (1983), Campbel e Beveridge (1994) Palm (1997). Os cálculos dos índices parasitários seguiram o trabalho de Serra-Freire (2002).

\section{Resultados}

Dos 201 peixes coletados, 138 estavam parasitados por plerocercos de cestoides da ordem Trypanorhyncha. As espécies identificadas foram: Poecilancistrium caryophyllum, Callitetrarhynchus gracilis, Pterobothrium heteracanthum e Pterobothrium crassicolle, e as prevalência ( $\mathrm{P} \%)$, intensidade média (IMP) e índice de abundância (IA), foram analisadas (Tabela 1).

Tabela 1: Índices parasitários encontrados nos scianideos estudados no litoral amazônico

\begin{tabular}{|c|c|c|c|c|c|c|}
\hline $\begin{array}{l}\text { Espécies de peixes } \\
\text { examinados }\end{array}$ & $\begin{array}{l}\text { № de peixes } \\
\text { examinados }\end{array}$ & $\begin{array}{l}\text { Quantidade } \\
\text { parasitada }\end{array}$ & $\begin{array}{c}\text { Espécies de parasitos } \\
\text { encontrados }\end{array}$ & $\mathrm{P}(\%)$ & IMP & ÍA \\
\hline M. ancylodon & 46 & 14 & P. caryophyllum & $30,43(14)$ & 2,28 & 0,69 \\
\hline P. squanosissimus & 45 & 32 & P. caryophyllum & $71,11(32)$ & 9.5 & 6.75 \\
\hline \multirow[t]{4}{*}{ C. acoupa } & 110 & 92 & C. gracilis & $60,0(66,0)$ & 3,82 & 2,36 \\
\hline & & & P. caryophyllum & $24,5(29,2)$ & 6,58 & 2,03 \\
\hline & & & P. heteracanthum & $40,0(47,8)$ & 13,35 & 5,82 \\
\hline & & & P.crassicolle. & $11,82(13,3)$ & 11,2 & 1,01 \\
\hline
\end{tabular}

econômicas às indústrias pesqueiras (São Clemente, 2007). Estes parasitos, quando adultos, têm como habitat o aparelho gastrintestinal de peixes elasmobrânquios. Suas formas larvares são encontradas em várias espécies de peixes teleósteos, elasmobrânquios e uma variedade de invertebrados marinhos (Campbell; Beveridge, 1994). O objetivo do presente trabalho foi analisar a presença de cestoides da ordem Trypanorhyncha, identificando as espécies e seus índices parasitários em scianideos comercializadas no estado do Pará.

\section{Material e métodos}

Durante o ano de 2006 e 2007 foram avaliados 201 exemplares de scianideos, distribuídos em: 110 exemplares de pescada amarela (Cynoscion acoupa, Lacepède, 1801); 45 exemplares de pescada branca (Plagioscion squanosissimus, Heckel, 1840); 46 exemplares de pescada gó (Macrodon ancylodon, Bloch e Scheider, 1801), oriundos de embarcações industriais e artesanais do município de Vigia de Nazaré, estado do Pará.

Os peixes foram adquiridos inteiros, resfriados, identificados e mensurados. Os filés foram obtidos através de uma incisão no sentido da região anal para região opercular e em seguida inspecionado em mesa de inspeção (candling table), considerando-se três regiões corporais: musculatura dorsolateral, musculatura ventro-abdominal e serosa abdominal parietal. A metodologia utilizada para coletar, fixar, corar, clarificar e montar os metacestoides foi de acordo com Amato
A espécie $P$. caryophyllum encontrava-se presente em todas as espécies de peixe estudadas, atingindo a maior prevalência na pescada branca $(71,11 \%)$. P. heteracanthum apresentou a maior intensidade média parasitária, seguida de $P$. crassicolle, enquanto $P$. caryophyllum apresentou a menor. Os maiores índices de abundância foram apresentados por $P$. caryophyllum na pescada branca e $P$.

heteracanthum, na pescada amarela. A pescada gó mostrou a menor intensidade média parasitária com também menor índice de abundância $(0,69)$. A pescada amarela apresentou infecções múltiplas por metacestoides.

\section{Discussão}

Plerocercos de $P$. caryophyllum, $P$. heteracanthum, $P$. crassicolle e $C$. gracilis têm sido registrados em teleósteos do litoral brasileiro, inclusive em scianideos, de várias regiões do Brasil (Palm, 1997; Pereira e Boeger, 2005; Oliveira et al., 2009; São Clemente et al., 2007), permitindo, deste modo, referenciar estes registros com os índices parasitários e locais de infecções encontrados no presente estudo. Porto et al. (2009) realizou coleta de 30 peixes de Micropogonias furnieri, provenientes do município de Niteroi, estado do Rio de Janeiro, onde o Trypanorhyncha encontrado apresentou prevalência de $26,7 \%$, intensidade média parasitária de 2,25 e índice de abundância 0,6, que são valores inferiores aos encontrados neste estudo, exceto para as espécies P.crassicolle e $P$. caryophyllum que apresentaram menor prevalência. Pereira Jr. e Boeger (2005) observaram que em 
180 peixes no município de Rio Grande, tinha prevalência de $66,7 \%$, intensidade média de 5,2 e abundância média de 0,17 , valores próximos aos encontrados neste estudo. $O$ parasitismo múltiplo encontrado na espécie C. acoupa corrobora com os achados por Palm (1997), Alves e Luque (2001), São Clemente et al. (2004) e São Clemente (2007).

Os locais de infecção encontrados apresentaram valores elevados, principalmente na musculatura de importância comercial, a região dorso-lateral. Estes índices parasitários aproximam-se com os registros de Amato et al. (1990) em K. pelamis na musculatura abdominal $(92,7 \%)$, Oliveira et al. (2009) em M. ancylodon na musculatura dorso-lateral $100 \%$ e $A$. proops na região abdominal com 95,24\%.

\section{Referências}

AMATO, J. R. F.; SÃO CLEMENTE, S. C.; OLIVEIRA, G. A. Tentacularia coryphaenae Bosc, 1801 (Eucestoda: Trypanorhyncha) in the inspection and technology of the skipjack tuna, Katsuwonus pelamis (L.) (Pisces: Scombridae). Atlântica. Rio Grande:v. 12, n. 1, p 73-77, 1990.

AMATO, J. F. R; BOEGER, W.A.; AMATO, S. B. Protocolos para laboratório - Coleta e processamento de parasitos de pescado. Imprensa Universitária - UFRRJ, p. 1-81, 1991.

ALVES, D. R. LUQUE, J. L. Aspectos quantitativos das infrapopu-lações de metazoários parasitos de Micropogonias furnieri (Osteichthyes: Sciaenidae) do litoral do estado do Rio de Janeiro, Brasil. Mem. Ins. Oswaldo Cruz, Rio de Janeiro, v. 25, n. 1-2, Santiago, ene. 2001.

CARVAJAL, J.; REGO, A. A. Progrillotia dollfusi sp. n. (Cestoda, Trypanorhyncha) parasito de pescado do Litoral Brasileiro. Men Inst. Oswaldo Cruz, Rio de Janeiro, v. 78, n. 2, p. 231-234, 1983.

CAMPBELL, R.A.; BEVERIDGE, I. Order Trypanorhyncha Diesing, 1863. In: KHALIL, L.F.; BRAY, R.A.; JONES, A. (Eds.). Keys to the cestode parasites of vertebrates. Cambridge: CAB International, 1994. Chapter 7, p. 51-148.

DOLLFUS, R. Ph. Études critiques sur les tétrarhynques du Muséum de Paris. Arch. Mus. Natl. Hist. Nat. Paris. n. 19, p. 1-466, 1942.

MENEZES, N. A.; FIGUEREDO, J. L. Manual de peixes marinhos do sudeste do Brasil. IV. Teleostei (3). São Paulo: Universidade de São Paulo, 1980.

OLIVEIRA, S. A. L; SÃO CLEMENTE. S. C. de; BENIGNO, R. N. M; KNOFF, M. Poecilancistrium caryophyllum (Diesing, 1850) (Cestoda Trypanorhyncha), parasito de Macrodon ancylodon (Bloch \& Schneider, 1801) do litoral Norte do Brasil. Rev. Bras. Parasitol. Vet., v. 18, n. 4, p. 71-73, 2009.

\section{Conclusões}

Cynoscion acoupa é de grande valor comercial e a mais susceptível a infecção por diferentes espécies de Trypanorhyncha. Entre as regiões do corpo dos peixes analisados, a musculatura dorso-lateral foi a que apresentou maior índice parasitário. Os valores de prevalência sugerem que $P$. caryophyllum é uma espécie que acomete facilmente $C$. acoupa, $M$. ancylodon e $P$. squanosissimus. O presente estudo mostrou a necessidade de se realizar estudos epidemiológicos das parasitoses em peixes de interesse comercial da região Amazônica, devido aos altos índices parasitários encontrados.

PEREIRA, J. J.; BOEGER, W. A. Larval Tapeworms (Platyhelminthes, Cestoda) from Sciaenid Fishes of the Southern Coast of Brazil. Zoosystema, v. 27, n. 1, p. 5-25, 2005. Publications Scientifiques du Muséum National d'Histoire naturelle, Paris. www.zoosystema.com.

PALM, W. H. Trypanorhynch Cestodes of commercial fishes from Northeast Brazilian Coastal Waters. Mem. Inst. Oswaldo Cruz, Rio de Janeiro, v. 92, n. 1, p. 69-79. 1997.

PORTO, C.J.S.;SÃO CLEMENTE, S. C.;FREITAS. M. Q.; SÃOCLEMENTE R. R. B.; KNOFF, M; MATOS, E. Pterobothrium crassicolle (Eucestoda: Trypanorhyncha) em corvinas, Micropogonias furnieri, comercializadas no município de Niterói, Rio de Janeiro, Brasil. $R$. bras. Ci. Vet., v. 16, n. 3, p. 133-135, set./dez. 2009.

RÊGO, A. A. Redescrição de Pterobothrium crassicolle Diesing, 1850 (Cestoda:Trypanorhyncha) e revalidação da espécie. Mem. Inst. Oswaldo Cruz. Rio de Janeiro: v. 82, n. 1, p. 51-53. 1987.

SÃO CLEMENTE, S. C.; KNOFF, M.; PADOVANI, R.E. S.; LIMA, F. C.; GOMES, D. C. Cestoides Trypanorhyncha Parasitos de Congro-Rosa, Genypterus Brasiliensis Regan, 1903 Comercializados nos Municípios de Niterói e Rio de Janeiro, Brasil. Rev. Bras. Parasitol. Vet., v.13, n. 3, p. 97-102, 2004.

SÃO CLEMENTE, S. C.; KNOFF, M.; LIMA, F. C.; ANDRADA, C. G.; FELIZARDO, N. N.; PADOVANI, R.E. S; GOMES, D. C. Cestóides Trypanorhyncha Parasitos de Peixe Sapo-Pescador, Lophius Gastrophysus Miranda-Ribeiro, 1915 Comercializados no Estado do Rio de Janeiro, Brasil. Rev. Bras. Parasitol. Vet., v.16, n. 1, p. 37-42, 2007.

SERRA-FREIRE, N. M. Planejamento e análise de pesquisas parasitológicas. Niterói: EdUFF, 2002. 